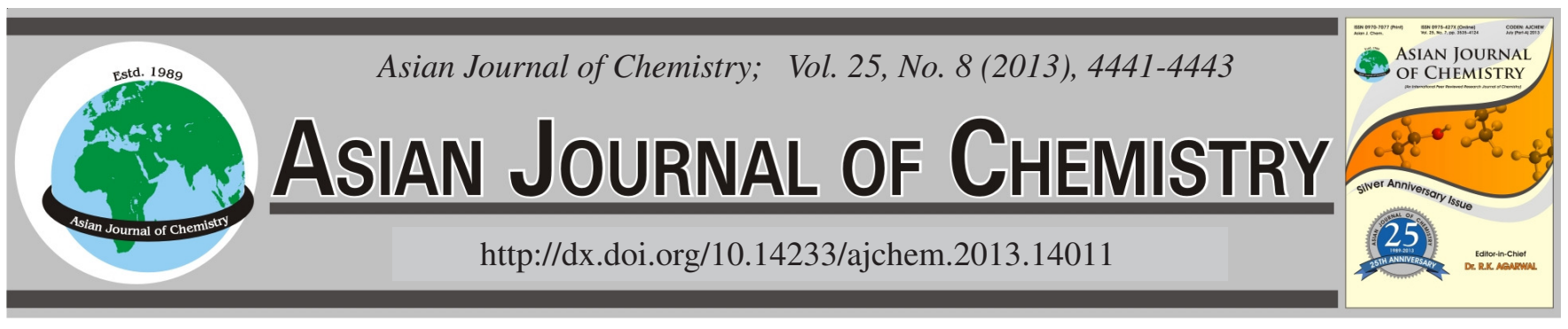

\title{
Esterification of tert-Butanol and Acetic Acid by Silicotungestic Acid Catalyst Supported on Bentonite
}

\author{
JIAN-ZHONG $\mathrm{JIN}^{*}$ and NA-Bo SuN
}

College of Biological and Environmental Engineering, Zhejiang Shuren University, Hangzhou, 310015, P.R. China

*Corresponding author: Fax: +86 571 88297098; Tel: +86 571 88297171; E-mail: hzjjz@ sohu.com

\begin{abstract}
A series of solid acid catalysts were synthesized by incipient wetness impregnation method by varying the wt $\%$ of silicotungstic acid on bentonite. Silicotungestic acid supported on bentonite was used to catalytic synthesis of tert-butyl acetate with acetic acid and tert-butyl alcohol. The main reaction parameters such as silicotungstic acid loading on bentonite, the amount of catalyst, molar ratio of reactants, reaction temperature and reaction time have been investigated. The optimum conditions were determined as follows: silicotungstic acid loading on bentonite $25 \mathrm{wt} \%$, catalyst $0.7 \mathrm{~g}$, mole ratio of tert-butanol to acetic acid $1: 1.1$, reaction temperature $110^{\circ} \mathrm{C}$ and reaction time $2 \mathrm{~h}$. The esterification yield of tert-butyl acetate was about $87.2 \%$. The catalyst could be used repeatedly for many times without distinct loss in activity.
\end{abstract}

Key Words: tert-Butyl acetate, Silicotungstic acid, Bentonite, Esterification.

\section{INTRODUCTION}

Esterification of alcohols, particularly normal alcohols, by carboxylic acids using homogeneous acid catalysts(viz. mineral acids) is well known ${ }^{1}$. However, it is extremely difficult to prepare tert-butyl ester by the esterification of tert-butanol using an acid catalyst. This is because of the high reactivity of tert-butanol in the presence of an acid, causing a rapid dehydration of the alcohol to iso-butylene, even at the room temperature ${ }^{2}$. Hence, there is a need to develop an environmentally benign method for the esterification of tert-butanol using a highly acitive, selective and reusable solid catalyst having a little or no acitivity for the dehydration of tertbutanol.

At present, the esterification reaction could be catalyzed by heterogeneous catalysts instead of homogeneous ones. Some heterogeneous catalysts reported include ion exchange resin, H-ZSM-5, zeolites-Y, niobic acid, sulfated oxides and SBA-15 supported heteropolyacids (HPA) ${ }^{3-6}$. They have many advantages, for example, they can be more easily separated and have higher catalytic activity.

In this work the silicotungestic acid catalyst supported on bentonite was employed in the synthesis of tert-butyl acetate. The main reaction parameters are silicotungestic acid loading on bentonite, the amounts of catalyst, molar ratio of reactants, reaction temperature and reaction time. Accurate control of these parameters is required in order to improve the yield of ester, hence, it is necessary to investigate systematically the effect of these reaction parameters. The optimum conditions were determined as follows: silicotungestic acid loading on bentonite $25 \mathrm{wt} \%$, catalyst $0.7 \mathrm{~g}$, mole ratio of tert-butanol to acetic acid $1: 1.1$, reaction temperature $110{ }^{\circ} \mathrm{C}$ and reaction time $2 \mathrm{~h}$. The esterification yield of tert-butyl acetate was about $87.2 \%$. The catalyst could be used repeatedly for many times without distinct loss in activity.

\section{EXPERIMENTAL}

Bentonite was obtained from Zhejiang Sanding Group Co. Ltd. and other reagents were of chemical grade and used without further purification.

The catalysts were prepared by wet impregnation method. A series of catalysts having different loading ranging from $10-30 \mathrm{wt} \%$ were synthesized by impregnating $5 \mathrm{~g}$ of bentonite with an aqueous solution of silicotungestic acid (STA) (30 $\mathrm{mL}$ of conductivity water) under constant stirring $6 \mathrm{~h}$ followed by heating till complete evaporation of water. It was dried in an oven at $110^{\circ} \mathrm{C}$ for $4 \mathrm{~h}$, then in miffle at $250^{\circ} \mathrm{C}$ for $5 \mathrm{~h}$.

Esterification reaction: The esterification reaction was carried out under batch reaction conditions using a $250 \mathrm{~mL}$ flask fitted with a water divider, a stirrer and a thermometer. The outlet of the water divider was connected to a reflux condenser. The temperature of the oilbath was fixed at the certain reaction temperature until reaction time was reached. A typical reaction mixture in the reactor contained acetic acid $(0.165$ 
$\mathrm{mol})$, tert-butanol $(0.15 \mathrm{~mol}), 10 \mathrm{~mL}$ toluene and the catalyst. In all cases, the reaction conversion ratio was determined by neutralization titration and calculated as follows:

$$
\text { Conversion }(\%)=\frac{A_{1}-A_{2}}{A_{1}} \times 100 \%
$$

where $A_{1}$ and $A_{2}$ were the acid values of the reactant mixture before and after the reaction, respectively.

\section{RESULTS AND DISCUSSION}

Influence of silicotungestic acid loading on bentonite: The influence of silicotungestic acid loading on bentonite on the acetic acid conversion was given in Fig. 1 under other identical reaction conditions. The silicotungestic acid loading on bentonite was varied from 10-30 wt \% while keeping the molar ratio of alcohol: acid at $1: 1.1$, catalyst $0.7 \mathrm{~g}$ and reaction temperature at $110{ }^{\circ} \mathrm{C}$. The reaction was carried out for $2 \mathrm{~h}$ and the products were analyzed. The conversion of acetic acid increases with silicotungestic acid loading on bentonite, reaching a maximum at $25 \mathrm{wt} \%$ silicotungestic acid and almost no changing thereafter.

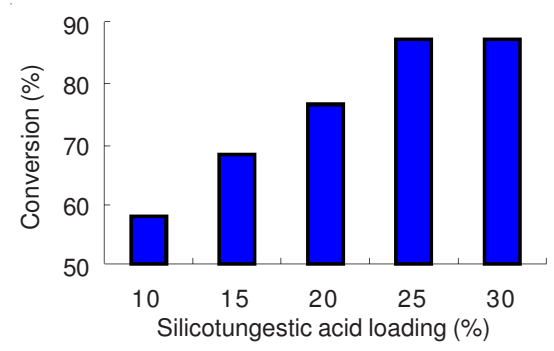

Fig. 1. Effect of silicotungestic acid loading on esterification of tertbutyanol and acetic acid carried out at reaction temperature $=110$ ${ }^{\circ} \mathrm{C}, n$-butanol:acetic acid $=1: 1.1$, time $=2 \mathrm{~h}$

Influence of catalyst amount: The amount of catalyst was varied from 0.3-0.8 g using $25 \mathrm{wt} \%$ silicotungestic acid while keeping the molar ratio of alcohol:acid at 1:1.1 and reaction temperature at $110{ }^{\circ} \mathrm{C}$. The reaction was carried out for $2 \mathrm{~h}$ and the products were analyzed. The results are represented in Fig. 2. With the increase in catalyst amount from 0.4-0.7 g, the conversion of acetic acid increases from 73.6$87.2 \%$ and almost no changing thereafter.

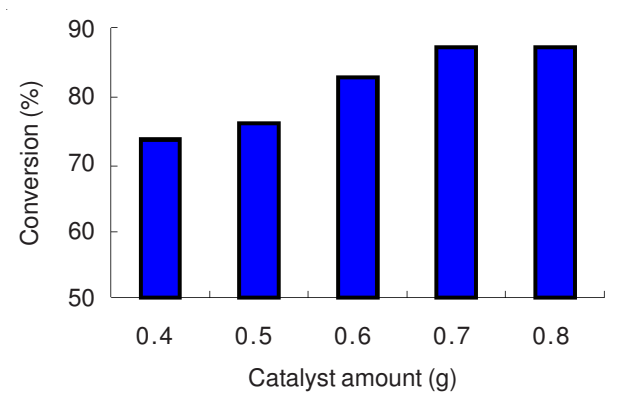

Fig. 2. Effect of catalyst amount on esterification of tert-butyanol and acetic acid carried out at reaction temperature $=110^{\circ} \mathrm{C}$, tert-butanol:acetic acid $=1: 1.1$, time $=2 \mathrm{~h}$

Influence of reaction temperature: Fig. 3 illustrates the effect of reaction temperature on the esterification of acetic

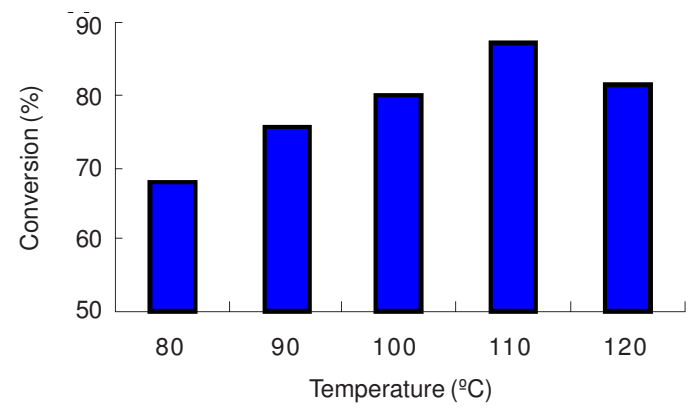

Fig. 3. Effect of reaction temperature on esterification of tert-butyanol and acetic acid using $25 \mathrm{wt} \%$ of silicotungestic acid as catalyst; catalyst amount $=0.7 \mathrm{~g}$, tert-butanol:acetic acid $=1: 1.1$, time $=2 \mathrm{~h}$

acid with $n$-butanol. The reaction was carried out in the temperature region $80-120^{\circ} \mathrm{C}$ taking $25 \mathrm{wt} \%$ silicotungestic acid as catalyst without altering other reaction parameters. The conversion increased from $67.5-87.2 \%$ with a change in reaction temperature from $80-110^{\circ} \mathrm{C}$. With further increase in reaction temperature, an increased conversion was observed.

Influence of molar ratio of reactants: Mole ratios of tert-butanol to acetic acid were varied from 1:0.9-1:1.3 and the result was shown in Fig. 4. In all cases and in all mole ratios of the reactants, tert-butyl acetate was observed as the main product. The conversion increased from $60.5-87.2 \%$ with a change in mole ratio of $n$-butanol to acetic acid from 1:0.9$1: 1.1$. With further increase in mole ratio of $n$-butanol to acetic acid, a slightly decrease in conversion was observed.

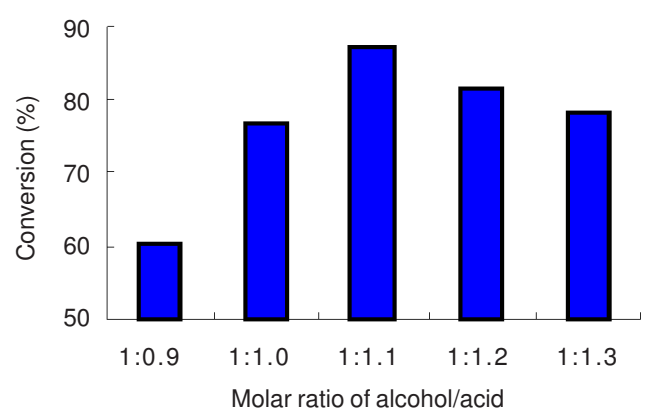

Fig. 4. Effect of molar ratio of alcohol/acid on esterification of tert-butyanol and acetic acid using $25 \mathrm{wt} \%$ silicotungestic acid as catalyst, catalyst amount $0.7 \mathrm{~g}$, reaction temperature $110{ }^{\circ} \mathrm{C}$, time $=2 \mathrm{~h}$

Influence of reaction time: The influence of reaction time on the acetic acid conversion was given in Fig. 5 using $25 \mathrm{wt}$ $\%$ silicotungestic acid as catalyst $(0.7 \mathrm{~g})$ under other identical reaction conditions. A gradual rise in the conversion was seen with increase in duration of the reaction period. As seen from Fig. 5 , in $2 \mathrm{~h}$ of reaction time, $87.2 \%$ of conversion is obtained, where as at the end of $2.5 \mathrm{~h}$ only $98.4 \%$ of the reaction is complete. This suggests that $2 \mathrm{~h}$ is sufficient to optimize the reaction parameters.

Reusability of the catalyst: The catalyst with $25 \mathrm{wt} \%$ loading was used for recycling experiments. In order to regenerate the catalyst after $2 \mathrm{~h}$ reaction, it was separated by filtration, washed with conductivity water several times, dried and calcined at $120^{\circ} \mathrm{C}$ and used in the esterification reaction with a fresh reaction mixture. In the regenerated sample after five cycles, the yield decreases by $5 \%$. 


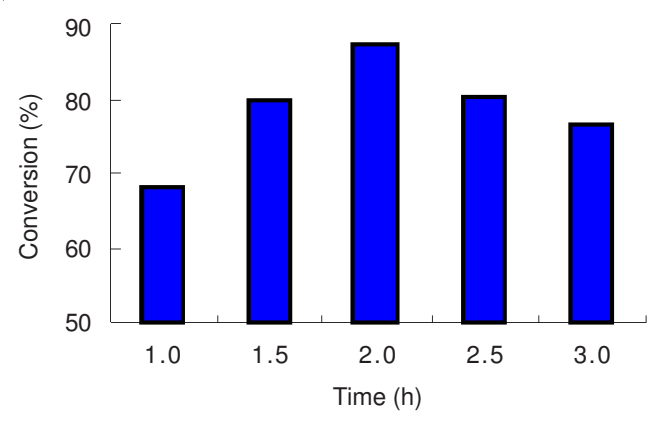

Fig. 5. Effect of reaction time on esterification of tert-butyanol and acetic acid using $25 \mathrm{wt} \%$ silicotungestic acid as catalyst, catalyst amount $=0.7 \mathrm{~g}, n$-butanol: acetic acid $=1: 1.1$

\section{Conclusion}

Silicotungestic acid (25 wt \%) supported on bentonite acts as an efficient and stable solid acid catalyst for esterification of tert-butyanol and acetic acid. The catalyst can be regenerated easily and reused at least five times.

\section{REFERENCES}

1. S. Patai, The Chemisty of Carboxylic Acid and Esters, Wiley, New York (1969).

2. V.R. Choudhary, K. Mantri and S.K. Jana, Catal. Commun., 2, 57 (2001).

3. S.S. Dash and K.M. Parida, J. Mol. Catal. A, 88, 266 (2007).

4. M. Misono, Catal. Rev. Sci. Eng., 29, 269 (1987).

5. P.G. Vazquez, M.N. Blanco and C.V. Caceres, Catal. Lett., 60, 205 (1999).

6. S. Swanmi, N. Shin-ichi, T. Okuhara and M. Misono, J. Catal., 166, 263 (1997) 